\title{
Die Schweizerische Gesellschaft für Biomedizinische Ethik: 20 Jahre Dialog zwischen Medizin und Gesellschaft
}

Samia Hurst

Prof. Dr. med.,

Ärztin und Bioethikerin

Präsidentin SGBE

\begin{abstract}
Die Medizin ist im grundlegenden Sinne des Wortes eine humanitäre Tätigkeit. Sie kommt mit der Intimität kranker Menschen in Berührung, tangiert Werte, die nicht selten in einem Spannungsverhältnis oder sogar Widerspruch zu alltäglichen Normen stehen. So erstaunt es wenig, dass sich in dieser Tätigkeit auch häufig ethische Probleme stellen, befinden wir uns doch in einem Bereich, der mit menschlichen Grenzerfahrungen zu tun hat und in dem Menschen arbeiten, denen die Ethik am Herzen liegt. Kommt dazu, dass bei medizinischen Dilemmaentscheidungen auch vernünftig begründete Meinungen durchaus auseinandergehen können.
\end{abstract}

\section{Gemeinsame Basis ist nicht dieselbe Ausbildung, sondern das Interesse an ethischen Problemen in der Medizin und den Biowissenschaften}

1 Callahan D. Bioethics as a discipline. In: Jecker NS, Jonsen AR, Pearlman RA, editors. Bioethics. Boston, London, Singapore: Jones and Bartlett; 1997.

2 Hurst S. La consultation d'éthique: à quoi ça sert et comment s'en servir? Revue Médicale Suisse. 2006;80:2195-9.

3 www.bioethica-forum.ch

Korrespondenz:

Prof. Samia Hurst Institut d'éthique biomédicale CMU

1 , rue Michel Servet

CH-1211 Genève 4

samia.hurst@unige.ch

www.bioethics.ch
Diese Umstände erklären zu einem guten Teil die Bedeutung der biomedizinischen Ethik. Von Beginn an hatte die Bioethik einige Schwierigkeiten zu überwinden. Entstanden ist sie in der Begegnung von klinisch Tätigen, die sich zusehends mit heiklen ethischen Entscheidungen konfrontiert sahen, mit Theoretikern der neuen Disziplin. Im Jahr 1973 kommentierte der Philosoph Daniel Callahan, einer der Pioniere und Gründer des Hasting Centers, diesen Neuaufbruch folgendermassen: «Ich wehrte mich panisch gegen die Idee, zusammen mit Ärzten deren Entscheidungen zu treffen. Ich? Da zog ich es eindeutig vor, den Ärzten schwierige Fragen zu stellen. In der Konfrontation mit realen Situationen wurde mir klar - und darin bestand meine Entschuldigung -, dass ich während meines Philosophiestudiums nichts gelernt hätte, was mich darauf vorbereitet hätte, zu einem bestimmten Zeitpunkt an einem bestimmten Nachmittag eine bestimmte ethische Entscheidung zu treffen. Ausgebildet war ich vielmehr in der klassischen Tradition philosophischer Reflexion, die sich durchaus bis zu zwei Jahrtausende Zeit zur Lösung schwerwiegender Probleme herausnahm.» [1]. Diesen Schwierigkeiten zum Trotz verlief die Begegnung gut und war für beide Seiten nützlich. Die Philosophen machten sich zusehends mit der Realität des medizinischen Alltags vertraut, während die Ärzte sich Zeit zum Studium der Ethiktheorie nahmen. Die ersten, aus unterschied- lichen Disziplinen stammenden Ethikberater wurden in den Spitälern integriert und deren Arbeit und neue Sichtweise als eine Bereicherung des klinischen Alltags empfunden [2].

Auch die vor genau 20 Jahren gegründete Schweizerische Gesellschaft für Biomedizinische Ethik (SGBE) hat diese Wurzeln. Sie versteht sich als ein interdisziplinäres Forum, in dem Probleme und Herausforderungen, die sich aufgrund des medizinischen Fortschritts, aber auch im klinischen Alltag ergeben, offen und lösungsorientiert diskutiert werden können. Mitglieder sind Ärztinnen, Philosophen, Pflegefachpersonen, Juristinnen, Theologinnen, Ethiker und andere. Jede interessierte Person kann bei uns Mitglied werden. Gemeinsame Basis ist nicht dieselbe Ausbildung, sondern das Interesse an ethischen Problemen in der Medizin und den Biowissenschaften. Gegründet wurde die SGBE im Bestreben, einen weltanschaulich offenen, interdisziplinären und demokratischen Austausch über die neuen Herausforderungen zu ermöglichen.

Um diese Ziele zu erreichen,

- veranstaltet die SGBE Seminare und Kolloquien $\mathrm{zu}$ ethischen Grundsatzfragen und ethisch umstrittenen Themen.

- veröffentlicht sie mit Bioethica Forum [3] eine interdisziplinäre und dreisprachige $(\mathrm{d} / \mathrm{f} / \mathrm{e})$ wissenschaftliche Zeitschrift (mit Peer-review), mit den Folia Bioethica eine Reihe mit kürzeren Arbeiten zu aktuellen Themen, mit dem SGBEbulletinSSEB einen thematischen Newsletter.

führt sie jährlich eine wissenschaftliche Tagung durch.

beteiligt sie sich an der Planung und Organisation von Aus- und Fortbildungskursen für Mitglieder von Ethikkommissionen.

\section{Jubiläums-Symposium}

Am 4. Dezember 2009 feiert die Schweizerische Gesellschaft für Biomedizinische Ethik ihr 20-jähriges Bestehen im Rahmen eines wissenschaftlichen Symposiums zum Thema «Die Zukunft der Bioethik». Bioethische Fragen werden sowohl in der klinischen Praxis als auch im Gesundheitssystem immer wichtiger. Das Symposium vom 4.12. im Inselspital Bern bietet eine gute Gelegenheit, die Bioethik mitsamt ihren Methoden, Themen und Auseinandersetzungen besser kennenzulernen. Programm unter www.sgbe-sseb.ch; Anmeldung unterinfo@bioethics.ch 